\title{
Wahlen mit System? Reformüberlegungen zur personalisierten Verhältniswahl*
}

\author{
Heiko Franke und Andreas Grimmel
}

Die Wahlen der Volksvertreter sind und bleiben das wichtigste und sensibelste Instrument in der repräsentativen Demokratie. Daher sind Fragen, die das Wahlverfahren und die Auswahl dieser Vertreter betreffen, von zentraler Bedeutung. Dementsprechend müssen in einer Demokratie Prozeduren gefunden werden, die im Hinblick auf Wahlen zweierlei leisten:

(1) Die Aufstellung der Kandidaten sollte die vielfältigen Interessen der Wähler widerspiegeln, so dass diese die Möglichkeit haben, durch ihre Entscheidung für eine Partei auch wirklich repräsentiert zu sein und nicht das Gefühl haben müssen, ihre Wahl mangels Alternativen getroffen zu haben. Dies schließt im Übrigen auch ein, dass jeder dafür qualifizierte Bürger eine Kandidatennominierung ohne größere Hürden anstreben kann.

(2) Der Output muss die Erwartungen an eine moderne und leistungsfähige Demokratie erfüllen. Denn was nützen noch so volksnahe Verfahren, wenn sie zu Ergebnissen führen, die den anerkannten Normen des Rechtsstaats widersprechen oder den politischen Willensbildungs- und Entscheidungsprozess zum Erliegen bringen? Dies ist eine Frage, die sich insbesondere die Befürworter jeglicher plebiszitärer Verfahren stellen müssen, und die im Hinblick auf die Erfordernisse eines parlamentarischen Regierungssystems nicht außer Acht gelassen werden darf.

Die parlamentarische Demokratie in Deutschland trägt diesen Erfordernissen mit der Ausgestaltung ihrer personalisierten Verhältniswahl allerdings nur unzureichend Rechnung: „Das Wahlsystem erfüllt zwar die Anforderungen der Parteienkonzentration gut, ist aber nur eingeschränkt repräsentativ. Partizipationschancen, Verständlichkeit und Transparenz fehlen weitgehend. Während das System bei dem Durchschnittsbürger nicht in Frage gestellt wird, ist es unter Experten der Wahlsystemanalyse, insbesondere Verfassungsjuristen, umstritten." 1 Das Statistische Bundesamt kommt in seinem Datenreport 2004 aufgrund der schwindenden Mitgliederzahlen der politischen Parteien zu einem Ergebnis ${ }^{2}$, das zum Nachdenken über die Ausgestaltung demokratischer Verfahrensweisen in Deutschland anregt: „Verliert die Mitgliedschaft in Interessengruppen und politischen Parteien für den Einzelnen an Attraktivität, so ist dies zunächst ein Warnsignal für die jeweilige Organisation. Geschieht dies jedoch in großem Umfang, weist es darüber hinaus auch auf generelle Probleme der Interessenvermittlung in einem politischen Gemeinwesen hin. “3

* Wir möchten unseren Beitrag Uwe Thaysen in Gedenken widmen.

1 Volker von Prittwitz, Vollständig personalisierte Verhältniswahl - Reformüberlegungen auf der Grundlage eines Leistungsvergleichs der Wahlsysteme Deutschlands und Finnlands, in: APuZ, B 52 (2003), S. 17.

2 Nach jüngsten Erhebungen des Statistischen Bundesamtes sind 1,6 Millionen Bundesbürger im wahlberechtigten Alter Mitglied in einer politischen Partei. Dies entspricht einem Anteil von 2,6 Prozent der Bevölkerung; vgl. Statistisches Bundesamt (Hrsg.), Datenreport 2004, 2. Auflage, Bonn 2005, S. 177.

3 Ebenda, S. 644. 
Hier soll ein Wahlsystem vorgestellt werden, das wirkungsvolle strukturelle Veränderungen beinhaltet, ohne jedoch den bewährten demokratischen Rahmen in Frage zu stellen. Es sollten sich durch die Reformvorschläge gerade hinsichtlich politischer Inhalte neue Möglichkeiten eröffnen, da bürgerschaftliches Engagement die Integration von bisher unzureichend genutztem Fachwissen fördern wird. Dabei erscheint es wichtig, dass einzelne Änderungsvorschläge nicht separat voneinander betrachtet werden, wie dies in zahlreichen Studien der Fall ist, sondern die einzelnen Teile ineinander greifen und aufeinander aufbauen.

\section{Die politischen Parteien}

Eines der Wesensmerkmale einer Demokratie liegt in der Begrenzung der Herrschaft auf eine gewisse Zeit. Die zentralen Verfassungsorgane müssen durch regelmäßig stattfindende und wiederkehrende Wahlen - Art. 39 Abs. 1 S. 1 GG gibt eine Wahlperiode von vier Jahren vor - demokratisch legitimiert sein. ${ }^{4}$ Die Wahlen erfüllen nur dann ihre Funktionen, die Politik zu lenken und zu kontrollieren ${ }^{5}$ sowie die öffentliche Meinung abzubilden, wenn den Bürgern sachliche und personelle Alternativen eröffnet werden. ${ }^{6}$

Das Volk muss das Ergebnis des öffentlichen Meinungsaustausches auch auf die sich zur Wahl stellenden, in aller Regel von den Parteien ${ }^{7}$ nominierten Repräsentanten übertragen können. Die Parteien entsprechen auch damit der von Art. 21 Abs. 1 S. 1 GG zuerkannten maßgeblichen Stellung bei der politischen Willensbildung des Volkes und üben unter anderem durch die gewählten Kandidaten Einfluss auf die staatliche Willensbildung aus. ${ }^{8}$ Parteien sind allerdings nicht nur für die Durchführung von Wahlen unerlässlich - und regelmäßig darauf folgend bei der Besetzung von Ämtern -, sondern sie sollen auch das Interesse und die Bereitschaft zur Partizipation der Bürger an politischen Vorgängen fördern, wie $₫ 1$ Abs. 2 PartG vorgibt. Fraglich ist allerdings, ob ihnen dies gelingt. Eine Quote parteipolitisch Engagierter von 2,7 Prozent im Westen und 1,8 Prozent im Osten Deutschlands ${ }^{9}$ spricht dagegen. Die geringe Partizipation dürfte nicht zuletzt Auswirkungen auf die Qualität der politischen Inhalte haben. Die Parteien und infolge dessen auch der Staat schöpfen das in der Gesellschaft vorhandene Potential nicht hinreichend aus. Im Sinne eines pluralistischen Gemeinwesens sollte möglichst vielen Ideen und Meinungen der Zugang zur Politik ermöglicht werden. Schließlich ist das politische System auf den Zufluss von Expertise angewiesen, um sich den in permanentem Wandel begriffenen Problemen zu stellen und Lösungen erarbeiten zu können.

4 Reinhold Zippelius / Thomas Würtenberger, Deutsches Staatsrecht, 31. Auflage, München 2005, S. 76.

5 Neben den Wahlen gibt es selbstverständlich intermittierende Kritik- und Kontrollmöglichkeiten im Bereich der öffentlichen Kommunikation. Dabei kann sich zum Teil ein enormer Druck auf das politische System von Seiten der Bürger entfalten. Politische Skandale bieten hierfür immer wieder anschauliche Beispiele; vgl. hierzu Sighard Neckel, Das Stellhölzchen der Macht. Zur Soziologie des politischen Skandals, in: Leviathan, 14. Jg. (1986), S. 581 - 605. Nicht zu vergessen sind auch die Ergebnisse politischer Meinungsumfragen.

6 Reinhold Zippelius / Thomas Würtenberger, a.a.O., S. 76.

$7 \$ 1$ Abs. 2 PartG: ,... sich durch Aufstellung von Bewerbern an den Wahlen in Bund, Ländern und Gemeinden beteiligen."

8 BVerfGE 20, S. 56, S. 101.

9 Zahlen nach: Statistisches Bundesamt (Hrsg.), a.a.O., S. 643. 
Das ist auch den Parteien bewusst, die sich zunehmend genötigt sehen, externe Experten zur Formulierung von Inhalten heranzuziehen. Diese Berater sind allerdings zum einen nicht direkt durch das Volk legitimiert. Zum anderen besteht die Gefahr, dass die Abgeordneten immer mehr zu bloßen Vermittlern oder Verwaltern als zu Gestaltern werden, wenn sie selbst zu wenig Kompetenz aufweisen. Weshalb Fach-Eliten trotz politischen Interesses ihren Weg nicht in die Parteien und somit auf dem herkömmlichen Weg in die Politik finden, liegt auf der Hand: Die Gelegenheit, für ein Amt aufgestellt zu werden beziehungsweise auf die aussichtsreichen Plätze der Parteilisten gewählt zu werden, ist oftmals an ein langwieriges und arbeitsintensives Parteiengagement geknüpft. Die Parteien sollten hieran arbeiten und kompetenten Bürgern den Einstieg in die Politik erleichtern. ${ }^{10}$ Es gilt, Sachverständigenkompetenz direkt wählbar zu machen, um eine kaum transparente „Expertokratie“ mit zweifelhafter Legitimation durch die Bürger zu vermeiden. ${ }^{11}$

\section{Die personalisierte Verhältniswahl}

In der Theorie konkurrieren das Mehrheitswahl- und das Verhältniswahlsystem. Das Grundgesetz selbst legt sich nicht auf ein Wahlsystem fest. Im Gegensatz zur Weimarer Reichsverfassung (Art. 22 Abs. 1 S. 1 WRV: Verhältniswahl) überlässt es dem Gesetzgeber die Entscheidung (Art. 38 Abs. 3 GG). ${ }^{12} \mathrm{Ihm}$ ist auch gestattet, beide Systeme miteinander zu verbinden. ${ }^{13}$ Davon wurde in Form der so genannten personalisierten Verhältniswahl Gebrauch gemacht. Danach wird die eine Hälfte der gegenwärtig vorgesehenen Anzahl von 598 Abgeordneten in den Wahlkreisen nach Kreiswahlvorschlägen gewählt, die andere Hälfte nach Landeswahlvorschlägen ( $\$ 1$ BWahlG). Jeder Wähler hat zwei Stimmen, eine Erststimme für die Wahl eines Wahlkreisabgeordneten und eine Zweitstimme für die Wahl nach Landeslisten der Parteien ( $\$ 4$ BWahlG). Mit der Erststimme wird gewählt, wer die meisten Stimmen innerhalb eines Wahlkreises auf sich vereint ( $\$ 5$ BWahlG). Nach dem Zweitstimmenanteil werden grundsätzlich die Sitze im Bundestag an die Parteien vergeben, wobei die von $\$ 6$ Abs. 6 S. 1 BWahlG vorgesehene Sperrklausel von fünf Prozent den Einzug sehr kleiner Gruppierungen verhindert. ${ }^{14}$

Diese eingeschränkte Verhältniswahl hat sich bewährt. Sie erscheint gerechter als das Mehrheitswahlsystem, das für eine Verzerrung zwischen dem Anteil an Wählerstimmen und den Kräfteverhältnissen im Parlament sorgen kann. ${ }^{15}$ Die Sperrklausel mag zwar mit dem Grundsatz der Wahlrechtsgleichheit schwer zu vereinbaren sein, wird aber für verfassungsgemäß gehalten, sofern sie nicht mehr als fünf Prozent beträgt ${ }^{16}$, und hat bisher für stabile Verhältnisse im Parlament gesorgt.

10 Vgl. Philip Zeschmann, Mitgliederbefragungen, Mitgliederbegehren und Mitgliederentscheide Mittel gegen Politiker- und Parteienverdrossenheit?, in: ZParl, 28. Jg. (1997), H. 4, S. 712 ff.

11 Vgl. auch Otmar Jung, Direkte Demokratie - Forschungsstand und Forschungsaufgaben 1995, in: ZParl, 26. Jg. (1995), H. 4, S. 674 ff.

12 BVerfGE 1, S. 208, S. 246; Reinhold Zippelius / Thomas Würtenberger, a.a.O., S. 347.

13 Reinhold Zippelius / Thomas Würtenberger, a.a.O., S. 347.

14 Zum Ausgleich sieht $\$ 6$ Abs. 6 S. 1 BWahlG die Grundmandatsklausel vor; dazu BVerfGE 95, S. 408, S. $423 \mathrm{f}$.

15 Hartmut Maurer, Staatsrecht I, 3. Auflage, München 2003, \$13 Rn. 25 (S. 397).

16 BVerfGE 51, S. 222, S. 236 f.; 82, S. 322, S. 338. 
Die Kombination von Zweitstimme und Erststimme lässt die personalisierte Verhältniswahl als Kompromiss zwischen (relativer) Mehrheitswahl und Verhältniswahl erscheinen. ${ }^{17}$ Bisweilen wird darin „ein hohes Maß an Persönlichkeitsbezogenheit" der Wahl erkannt. ${ }^{18}$ Es muss aber bezweifelt werden, dass für die Wähler die Persönlichkeit des Abgeordneten im Vordergrund steht. Vielmehr kennen sie oftmals die mit der Erststimme wählbaren Direktkandidaten nicht und orientieren sich am politischen Programm der hinter den aufgestellten Kandidaten stehenden Parteien ${ }^{19}$ beziehungsweise nehmen ein so genanntes Stimmensplitting vor ${ }^{20}$, das heißt, sie geben ihre Erststimme dem Kandidaten einer Partei, die sie mit ihrer Zweitstimme nicht unterstützen. Davon sind regelmäßig die kleinen Parteien betroffen. CDU/CSU und SPD vereinigen mit wenigen Ausnahmen alle erfolgreichen Direktkandidaten auf sich, während der Erststimmenanteil der kleinen Parteien gegenüber dem Zweitstimmenanteil abfällt. So errangen die Grünen bei der Bundestagswahl 2002 8,6 Prozent der Zweitstimmen, erhielten aber nur 5,6 Prozent der Erststimmen. ${ }^{21}$

Dies dürfte nicht darauf zurückzuführen sein, dass die Wähler das Personal der kleineren Parteien als vergleichsweise weniger kompetent wahrnehmen. Vielmehr wird der Grund darin liegen, dass die Vergabe der Erststimme an den Kandidaten einer kleinen Partei von vornherein aussichtslos erscheint und die Wähler ihre Stimme nicht „verschenken “22 wollen. Die Wahl reduziert sich also faktisch stark auf CDU/CSU- und SPD-Kandidaten, ist folglich in erheblichem Maße vorselektiert und fördert taktisches Stimmverhalten anstelle des ursprünglich gewollten. ${ }^{23}$ Davon allerdings, so muss hinzugefügt werden, profitieren die kleinen Parteien auch, wenn sie Listenstimmen als „Leihstimmen“ ihres großen Koalitionspartners erhalten.

Es ist aber auch möglich, dass die Wähler das Wahlsystem nicht hinreichend verstehen. Rüdiger Schmitt-Beck wies bereits 1993 darauf hin, dass sich „die pessimistischen Diagnosen früherer Studien über die verbreitete Unkenntnis der Wähler bezüglich des Modus der Mandatsverteilung im Bundestag, der mit dem Zwei-Stimmen-Wahlsystem verbunden ist “ 24 , bestätigt hätten. So führe das so genannte Stimmensplitting zu Verzerrungen des

17 Reinhold Zippelius / Thomas Würtenberger, a.a.O., S. 347.

18 So ebenda.

19 Eckhard Jesse, Reformvorschläge zur Änderung des Wahlrechts, in: APuZ, B 52 (2003), S. 3, S. 6; Claus Arndt, Fraktion und Abgeordneter, in: Hans-Peter Schneider / Wolfgang Zeh (Hrsg.), Parlamentsrecht und Parlamentspraxis, Berlin / New York 1989, S. 646, Rn. 8.

20 Eckhard Jesse, Split-Voting in the Federal Republic of Germany: An Analysis of the Federal German Elections from 1953 to 1987, in: Electoral Studies, 7. Jg. (1988), S. 109 - 124; ders., a.a.O. (2003), S. 3, S. 6.

21 ders., a.a.O. (2003), S. 3, S. 6.

22 Ebenda. Jesse weist allerdings darauf hin, dass dies empirisch nicht fundiert beantwortet werden kann.

23 So wählten bei der Bundestagswahl 2002 etwa 60 Prozent derjenigen, die mit ihrer Zweitstimme Bündnis 90/Die Grünen, mit ihrer Erststimme aber den Bewerber einer anderen Partei unterstützten, einen SPD-Kandidaten, vgl. Statistisches Bundesamt (Hrsg.), Wahl zum 15. Deutschen Bundestag am 22. September 2002, H. 4/2003, S. 20. Da SPD und Grüne zu jener Wahl als vorherige Koalitionspartner antraten, musste die Abgabe einer Erststimme an einen SPD-Kandidaten nicht verloren erscheinen.

24 Rüdiger Schmitt-Beck, Denn sie wissen nicht, was sie tun ... Zum Verständnis des Verfahrens der Bundestagswahl bei westdeutschen und ostdeutschen Wählern, in: ZParl, 24. Jg. (1993), H. 3, S. 414. 
Wählerwillens. ${ }^{25}$ Dieses Strukturproblem könnte bei der Bundestagswahl 2005 aufgrund der knappen Mehrheiten entscheidend gewesen sein.

Es ist somit festzustellen, dass die Erststimme von den Wählern oft nicht zweckgemäß genutzt wird. Ferner ist zu kritisieren, dass sich lediglich ein einziger Kandidat pro Partei, der gemäß $₫ 21$ Abs. 1 S. 1 BWahlG parteiintern aufgestellt worden ist, um ein Direktmandat bewirbt. Das hat zur Folge, dass er sich bei der Bundestagswahl nicht gegenüber einem anderen Mitglied derselben Partei durchsetzen muss und nicht gezwungen ist, seine Persönlichkeit und politischen Anliegen eingehend vorzustellen, sondern auf die Programmatik seiner Partei verweisen kann. Dabei wäre eine größere Auswahl zwischen Personen, die im Kern dieselbe politische Richtung vertreten, im Sinne einer echten Entscheidung für eine Person und die von dieser propagierten Politikinhalte wünschenswert.

Darüber hinaus gibt die Erststimme den Wählern häufig nicht die Möglichkeit, sich effektiv gegen eine Person auszusprechen, indem sie eine andere wählen, denn oft ziehen die erfolglosen Kandidaten über die Landesliste in den Bundestag ein, so dass - neben den oben angeführten Mängeln - auch Ineffizienz zu beanstanden ist. ${ }^{26}$

Alles in allem erscheint das Zwei-Stimmen-Wahlrecht in seiner gegenwärtigen Ausprägung verbesserungsbedürftig. Dieser Eindruck wird durch einen weiteren Nachteil verstärkt: Durch die Trennung der zwei Stimmen bei Berücksichtigung der durch jede Landesliste erzielten Ergebnisse kommt es zu einem zwar verfassungsrechtlich zulässigen ${ }^{27}$, aber im Sinne des Proporzes nicht wünschenswerten Phänomen, den so genannten Überhangmandaten $^{28}$. Übersteigen die in den Wahlkreisen mit der Erststimme errungenen Sitze die Zahl der nach den Zweitstimmenanteilen errungenen, so verbleiben sie doch der Partei gemäß $\$ 6$ Abs. 5 BWahlG. Das hat zur Folge, dass die begünstigte Partei weniger Stimmen für ein Mandat im Bundestag benötigt als die anderen Parteien. Damit wird die vom Prinzip der Wahlrechtsgleichheit gebotene Sitzverteilung im Bundestag missachtet ${ }^{29}$ und das Wahlsystem intransparenter.

\section{Das Personen-Verhältnis-Wahlsystem als mögliche Alternative}

In Diskussionen um die Stärkung der Partizipation des Bürgers am politischen Willensbildungs- und Entscheidungsprozess werden nicht selten plebiszitäre Modelle wie Volksbefragung und Volksentscheid vorgeschlagen. Dabei wird oft übersehen, dass solche Verfahrensweisen den Anforderungen einer modernen Demokratie nicht gerecht werden können. Die Kritik muss sich hier vor allem an den zu erwartenden Ergebnissen entzünden, die nicht

$25 \mathrm{Vgl}$. ebenda, S. 415.

26 Eckhard Jesse, a.a.O. (2003), S. 3, S. 6.

27 BVerfGE 95, S. 335, S. 357 ff.; auf diese Problematik kann hier nicht näher eingegangen werden.

28 Vgl. dazu unter anderem David N. Rauber, Überhangmandate - keine Überraschungen (mehr), in: ZParl, 34. Jg. (2003), H. 1, S. 116 ff.; Helmut Nicolaus, Stimmgewicht und Erfolgschancengleichheit im Wahlverfassungsrecht. Prinzipielle Bemerkungen zur Zählwertgleichheit und zur Inflation der Überhangmandate, in: ZParl, 26. Jg. (1995), H. 2, S. 353 ff.; Waldemar Schreckenberger, Zum Streit über die Verfassungsmäßigkeit der Überhangmandate, in: ZParl, 26. Jg. (1995), H. 4, S. $678 \mathrm{ff}$.

29 Reinhold Zippelius / Thomas Würtenberger, a.a.O., S. 349. 
zwangsläufig mit den allgemein anerkannten Grundsatzentscheidungen des Rechtsstaats übereinstimmen müssen. Möchte man auch aus diesen Gründen das bewährte System der Repräsentation beibehalten, so müssen Reformen bei den Wahlen und Parteien ansetzen, denen eine zentrale Rolle bei der Gestaltung und Umsetzung von Politik zukommt. Doch auch die Parteien sehen sich mit großen Problemen konfrontiert: Rückgang der Mitgliederzahlen, Überalterung und Defizite in der innerparteilichen Partizipation. ${ }^{30}$ Dass die Parteien um diese Probleme wissen, geht aus immer wieder geführten Reformdebatten hervor. So bemühte sich zum Beispiel der seinerzeitige Generalsekretär der SPD, Franz Müntefering, mit eingängigen Formeln wie „30 unter 40“, „Zehn von Außen“ und der Forderung nach Vorwahlen bei der Kandidatenaufstellung für die Bundestagswahl, neue Zugangsmöglichkeiten für Interessierte zu schaffen. ${ }^{31}$

Damit die Politik auf die brachliegenden personellen und inhaltlichen Ressourcen zurückgreifen kann und zugleich eine größere Legitimationsbasis für ihre Entscheidungen erhält, sollten vor allem einige Reformen des Wahlsystems in Betracht gezogen werden. Dabei sind nur Modifikationen der bisherigen Regeln erforderlich. Sie betreffen die Kandidatenaufstellung, die Parteimitgliedschaft und das Wahlverfahren. Im Ergebnis sollte eine Öffnung der politischen Sphäre erzielt werden, ohne dass die Grundpfeiler des Parlamentarismus, insbesondere das sensible Fraktionsgefüge, geschwächt werden.

\subsection{Kandidatenaufstellung}

Die Aufstellung der Kandidaten umfasst Findung und förmliche Nominierung des Kandidaten. ${ }^{32}$ Beides sind parteiinterne Aufgaben, die für den Wähler meist undurchsichtig bleiben. So sieht er sich genötigt, einem ihm in der Regel unbekannten Parteikandidaten zuzustimmen.

Um eine stärkere Bindung zwischen Wählern und Kandidat herzustellen und dessen Aufstellung transparenter und demokratischer zu gestalten, wird hier die Einführung von Vorwahlen innerhalb der Parteien vorgeschlagen. ${ }^{33}$ Durch einen offeneren Kandidatenwettbewerb fänden kreative Politikinhalte ihren Weg schneller als bisher in die Politik. ${ }^{34}$ Auch die Findung der Kandidaten könnte in diesem Rahmen erfolgen. Für sie würde dann bereits im Vorfeld geworben, und die Kandidaten würden bekannter als bisher. Dies erfordert jedoch eine Öffnung der Parteien nach außen, welche sich vor allem durch flexiblere Parteimitgliedschaften erreichen ließe.

30 Vgl. Andreas Kießling, Politische Kultur und Parteien in Deutschland - Sind die Parteien reformierbar, in: APuZ, B 10 (2001), S. 29.

31 Vgl. Franz Müntefering, Demokratie braucht Partei - Die Chance der SPD, in: ZParl, 31. Jg. (2000), H. 2, S. $341 \mathrm{f}$.

32 Vgl. zum derzeitigen Stand der Forschung jüngst Suzanne S. Schüttemeyer / Roland Sturm, Der Kandidat - das (fast) unbekannte Wesen: Befunde und Überlegungen zur Aufstellung der Bewerber zum Deutschen Bundestag, in: ZParl, 36. Jg. (2005), H. 3, S. 539 ff.

33 Zur Einführung von Vorwahlen in Deutschland vgl. auch Sven T. Siefken, Vorwahlen in Deutschland? Folgen der Kandidatenwahl nach U.S.-Vorbild, in: ZParl, 33. Jg. (2002), H. 3, S. 531.

34 Vgl. Gerd Mielke, Mehr Demokratie wagen! SPD-Führung im partizipatorischen Zeitalter, in: Blätter für deutsche und internationale Politik, 42. Jg. (1997), S. 47. 


\subsection{Flexibilisierung der Parteimitgliedschaft}

Die Mitwirkung in einer Partei und somit auch die Entscheidungen über personelle Fragen sind in Deutschland an die Parteimitgliedschaft geknüpft. Diese erfordert neben Zeit zur Mitarbeit und Geld für die zum Teil nicht unbeträchtlichen Mitgliedsbeiträge immer auch eine sichere Überzeugung von den Parteiinhalten. Ausweislich der sinkenden oder bestenfalls stagnierenden Mitgliederzahlen ${ }^{35}$ besteht heute nur noch ein geringes Interesse von Seiten der Bürger an einer solchen Mitgliedschaft. Zur Einführung von Vorwahlen und offener Kandidatennominierung erscheint eine Art Parteiregistrierung neben der herkömmlichen Mitgliedschaft sinnvoll. Diese könnte die Voraussetzung für die Teilnahme an Vorwahlen und für die eigene Nominierung durch eine gewisse Anzahl der Parteianhänger beinhalten. Nach dem Prinzip der aus den USA bekannten so genannten Closed Primaries müsste sich jeder, der an den Vorwahlen teilnehmen möchte, circa zwölf Monate zuvor durch Registrierung einer Partei zuordnen. Ein Parteiwechsel wäre nur in einer gewissen Frist zwischen ein bis zwei Jahren zulässig. ${ }^{36}$ Eine Registrierung hätte den Vorteil, mehr Menschen als bisher mit den Parteien in Kontakt zu bringen und vor allem einen offeneren Wettbewerb der Kandidaten auszulösen, weil sich diese stärker den Interessen und Wünschen der Wähler anpassen müssten. Die zur Wahl stehenden Repräsentanten würden sich nicht erst im Wahlkampf darum bemühen, ihre Politikziele darzustellen. So dürften die Wähler sich wieder mehr durch die Politik angesprochen fühlen und eine größere Bindung zu den Parteien entwickeln. ${ }^{37}$

\subsection{Wahlverfahren}

Um die genannten Schwachpunkte des bisherigen Wahlverfahrens zu beheben, sollten Erstund Zweitstimme strikter voneinander getrennt werden, indem beide Stimmen separat ausgewertet und nicht miteinander verrechnet würden. Ein Teil der Parlamentssitze würde an die Direktkandidaten vergeben, ein anderer an die über die Zweitstimmen einziehenden Abgeordneten (vgl. Abbildung 1). Das hätte die Konsequenz und den Vorteil, dass die Direktwahl der Kandidaten auch als solche wahrgenommen würde und nicht nur als Ergänzung der Zweitstimme; ferner entfiele das Phänomen der Überhangmandate. Eine Erhöhung der Transparenz und Verständlichkeit von Wahlen wäre die Folge.

Zudem erscheint im Interesse einer modernen Anpassung des Wahlsystems auch das Kumulieren und Panaschieren von Wert zu sein. ${ }^{38}$ Das System ermöglicht dem Bürger,

35 Vgl. zum letzten Stand der Mitgliederzahlen Oskar Niedermayer, Parteimitgliedschaften im Jahre 2006, in: ZParl, 38. Jg. (2007), H. 2, S. 368 ff.

36 Vgl. hierzu auch Fritz Plasser, Vorwahlen und Vorwahlpraxis in den Vereinigten Staaten, in: Heinrich Neisser / Fritz Plasser (Hrsg.), Vorwahlen und Kandidatennominierung im internationalen Vergleich, Wien 1992, S. $12 \mathrm{ff.}$

37 Vgl. Philip Zeschmann, a.a.O., S. 711.

38 Vgl. Jürgen Dittberner, Die deutschen Parteien - Defizite und Reformideen, in: APuZ, B 40 (2004), S. 18; zur Diskussion um das Hamburger Wahlrecht vgl. Frank Decker, Parlamentarische Demokratie versus Volksgesetzgebung. Der Streit um ein neues Wahlrecht in Hamburg, in: ZParl, 38. Jg. (2007), H. 1, S. 118 ff. Zu den Erfahrungen mit Kumulieren und Panaschieren bei Kommunalwahlen vgl. Paul Tiefenbach, Kumulieren, Panaschieren, Mehrmandatswahlkreise - mehr Demokratie beim Wahlrecht?, in: ZParl, 37. Jg. (2006), H. 1, S. 115 ff. 


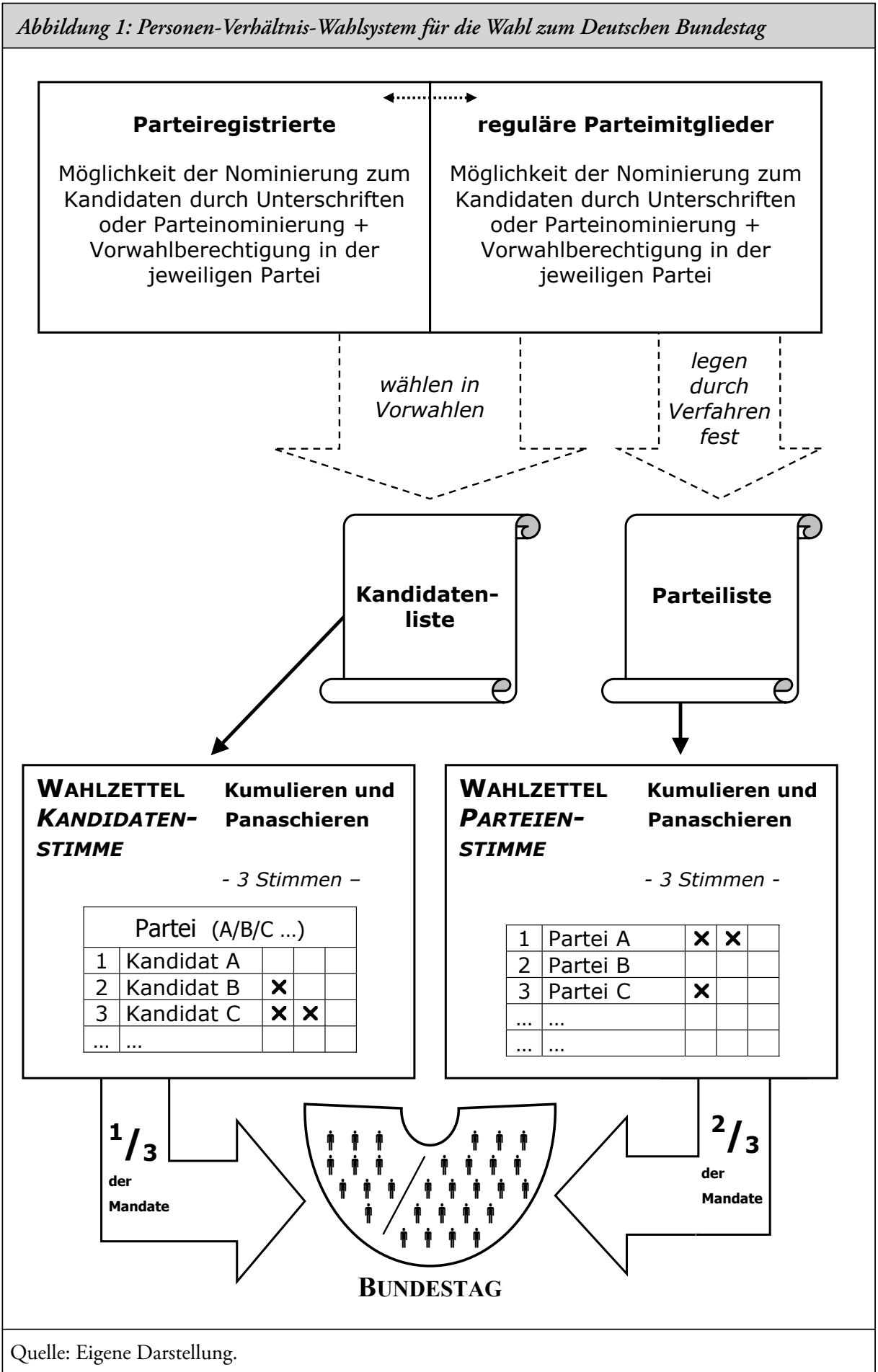


seine Präferenzen genauer zu artikulieren, als das zurzeit der Fall ist. Dazu bedürfte es lediglich der Erweiterung von je einer (geltendes Wahlsystem) auf je drei Stimmen (PersonenVerhältnis-Wahlsystem) für Direktmandat (Erststimme) und Parteiliste (Zweitstimme). Diese Stimmen ließen sich dann frei auf die Kandidaten beziehungsweise Parteien verteilen. Auf diese Weise hätte der Wähler die Möglichkeit, sehr viel genauere Aussagen über seine Präferenzen bezüglich der Besetzung politischer Ämter zu machen und in gewissem Maße - nämlich mit der Zweitstimme - sogar Koalitionswünsche zu äußern, ohne dies wie bisher zweckwidrig und verzerrend mit der Erststimme tun zu müssen.

Im Fall der Erst- beziehungsweise Kandidatenstimme bietet es sich an, dass nunmehr nicht nur derjenige mit den meisten Stimmen ein Mandat erlangt, sondern die drei Kandidaten mit den meisten Stimmen des Wahlkreises, so dass reelle Chancen auch für die Kandidaten kleiner Parteien bestehen, Direktmandate zu erlangen. Bei diesem System ist zu erwarten, dass die großen Parteien zwei bis drei und die kleineren ein bis zwei Kandidaten aufstellen, um eine Kumulation der Stimmen auf ihre Kandidaten zu ermöglichen. Bei dem so entstehenden auch innerparteilichen Wettbewerb wird genau zu überlegen sein, welche Kandidaten die Wünsche der Wähler treffend repräsentieren und folglich wählbar sind. Auch hier leisten die Vorwahlen ein gutes Stimmungsbarometer im Vorfeld der eigentlichen Wahl. Im Ergebnis wird der einzelne Abgeordnete und damit auch das Freie Mandat gestärkt, ohne die für ein handlungsfähiges Parlament unverzichtbaren Fraktionen zu schwächen. Er kann sich - eher als bisher - als selbst gewählt wahrnehmen, weil er ein eigenes Programm zur Wahl gestellt hat, das sich zwar im Rahmen des Parteiprogramms bewegt, jedoch individuelle Akzente setzt. Gegen eine völlige Abkehr von den Programminhalten der Partei verbleibt dieser als ultima ratio der Partei- beziehungsweise Fraktionsausschluss.

Durch dieses Verfahren würde eine Vergrößerung der Wahlkreise bei Verringerung ihrer Anzahl nötig; denn erhöhte man die Zahl der Direktkandidaten von einem auf drei, so bliebe nur die Maßnahme einer Wahlkreisverringerung, wenn nicht der ohnehin schon große Bundestag weiter anwachsen soll. Ein Problem aus der notwendigen Neuordnung der Wahlkreise könnte hierbei entstehen, weil der einzelne Abgeordnete fortan für mehr Bürger als bisher zuständig wäre. Eine gewisse Bürgernähe könnte hierbei der Politik abhanden kommen. Dem entgegenzustellen ist die Tatsache, dass die Abgeordneten im Rahmen des vorgeschlagenen Personen-Verhältnis-Wahlsystems stärker als bisher um die Stimmen der Bürger in ihrem Wahlkreis kämpfen müssten. Die verstärkte Personalisierung dürfte eine Intensivierung von Öffentlichkeitsarbeit und eine stärkere und direktere Orientierung an den Bedürfnissen der Bürger in den Wahlkreisen - auch außerhalb des Wahlkampfes - zur Folge haben.

Alles in allem entsteht auf diese Weise ein Wahlsystem, das den von Dieter Nohlen formulierten Anforderungen ${ }^{39}$, nämlich Repräsentation, Partizipation, Konzentration, Einfachheit und Legitimität Rechnung trägt.

Repräsentation: Insbesondere durch die Einführung von Vorwahlen und Kumulieren / Panaschieren gewinnen die Bürger größeren Einfluss auf die Auswahl der politischen Repräsentanten. So könnten sie genauer artikulieren, welche Kandidaten und somit auch welche Inhalte sie vertreten sehen möchten. Damit würde dem Grundsatz der Unmittelbarkeit der Wahl stärker entsprochen. Gleichzeitig bliebe den Parteien über die Parteilisten die Möglichkeit, ergänzende Personalnominierungen vorzunehmen, um einer zu einseitigen

39 Vgl. Dieter Nohlen, Wahlrecht und Parteiensystem, 3. Auflage, Opladen 2000, S. 157 ff. 
Kandidatenaufstellung vorzubeugen. Denkbar wäre hier zum Beispiel ein Verhältnis von ein Drittel (Direktkandidaten per Vorwahl) zu zwei Dritteln (Kandidaten der Parteilisten), welche in den Bundestag einziehen. ${ }^{40}$

Partizipation: Neben der Gelegenheit, sich schon vor, aber auch bei der eigentlichen Wahl für oder gegen bestimmte Kandidaten auszusprechen und damit aktiv an der Entscheidung über Personalfragen teilzunehmen, besteht bei Realisierung des Reformvorschlags die Chance, qualifizierten Quereinsteigern den Weg in die Politik zu ebnen. Ein großes, unzureichend genutztes Reservoir von Expertenwissen könnte Eingang in die Parteien finden. Zu oft wird heute die Möglichkeit, politische Verantwortlichkeit zu übernehmen, an eine lange, für viele unattraktive Parteikarriere gebunden und so der Zustrom kreativen Wissens gehemmt. ${ }^{41}$ Dies gilt nicht nur für kommunale Parteiämter, sondern im Prinzip für alle Repräsentativfunktionen bis hin zum Bundestagsmandat.

Konzentration: Das Kriterium der Konzentration zielt auf die Eigenschaft von Wahlsystemen, stabile Regierungsmehrheiten zu fördern, um die Handlungsfähigkeit der Politik zu gewährleisten. In dieser Hinsicht dürfte der Reformvorschlag keine Schwächung gegenüber dem geltenden Wahlrecht bedeuten. Ein wesentlicher Faktor, der bisher in der Bundesrepublik instabile Verhältnisse verhindert hat, ist die beizubehaltende Fünf-Prozent-Sperrklausel. Eine ernste Gefahr, dass radikale, die freiheitlich-demokratische Grundordnung nicht respektierende Parteien bessere Ergebnisse als bisher erzielen könnten, da bei drei zu vergebenden Stimmen pro Wähler häufiger auch eine an solche Parteien vergeben werden könnte, dürfte nicht bestehen. Ebenso könnte das Gegenteil der Fall sein, wenn sich nämlich die Wähler mehr mit dem politischen System und den etablierten Parteien beziehungsweise Parteipolitikern identifizieren als zuvor, da sie größere Partizipationsmöglichkeiten erhalten. Außerdem muss jede Demokratie mit solchen Kräften umgehen können, da sie eine notwendige Folge des Pluralismus sind. Die demokratischen Parteien und Abgeordneten haben darauf in der politischen Auseinandersetzung entsprechend zu reagieren. Über die Zweitstimme wird es also nicht zu einer Destabilisierung kommen. Aus den gleichen Gründen ist dies auch nicht bezüglich der Erststimme zu befürchten. Da somit davon auszugehen ist, dass eine überaus große Zahl der Abgeordneten nach wie vor von demokratischen Parteien gestellt werden wird, dürfte es keine Schwierigkeiten geben, stabile Regierungsmehrheiten zu finden.

Einfachheit: Ein großer Vorzug des Personen-Verhältnis-Wahlsystems liegt in seiner größeren Verständlichkeit. Jeder Bürger bekäme drei Erststimmen, die er Personen, und drei Zweitstimmen, die er Parteien zuordnen kann. Die tatsächlich weit verbreiteten Verwirrungen bezüglich der Bedeutung und Verrechnung der beiden Stimmen würden sich auflösen, da eine strikte Trennung in Personen-Stimme und Parteien-Stimme erfolgte und die Sitze im Bundestag strikt getrennt nach den jeweiligen Ergebnissen vergeben würden. Vom bisherigen System geförderte, obgleich unerwünschte Phänomene wie das Stimmensplitting wären hinfällig. Nunmehr könnten die Bürger sogar, sofern sie es wünschten, tatsächlich verschiedene Parteien wählen und damit eine Koalitionsaussage treffen.

Legitimität: Mit dem hier vorgeschlagenen Wahlsystem verbindet sich erstens die Hoffnung, dass die flexiblen und weitreichenden Mitbestimmungsmöglichkeiten eine große

40 Von diesem Verhältnis soll ausgegangen werden, denkbar ist aber auch eine hälftige Verteilung.

41 Vgl. Dietmar Herz, Politiker, in Beton gegossen, in: Die Zeit, http://zeus.zeit.de/text/ 2002/28/200228_eliten_xml, 2002. 
Akzeptanz durch die Bürger genießen und an vielen Stellen zum Mitmachen anregen werden. Zweitens dürfte sich ein großer Gewinn vor allem durch die steigende Transparenz politischer Entscheidungen ergeben, wenn es tatsächlich gelingen sollte, aus einem breiteren Spektrum der Bürgerschaft Kandidaten zu rekrutieren. Dann wären möglicherweise die politischen Repräsentanten weniger auf Fachwissen von „außen“ angewiesen, sondern könnten es direkt aus ihren eigenen - demokratisch gewählten - Reihen beziehen.

\section{Gegenargumente und Erfolgsaussichten}

Einwände gegen das Personen-Verhältnis-Wahlsystem werden sich kaum auf die verfassungsrechtliche Zulässigkeit beziehen. Das Bundesverfassungsgericht hat klargestellt, dass das Grundgesetz dem Gesetzgeber einen breiten Entscheidungsspielraum eröffnet, solange die Gleichheit der Wahl im jeweiligen Teilwahlsystem gewahrt werde, die Systeme sachgerecht zusammenwirkten und Unmittelbarkeit und Freiheit der Wahl nicht gefährdet würden. ${ }^{42}$ Anderes ist in Bezug auf den Vorschlag zu erwarten, Vorwahlen einzuführen. Gegen diese wird häufig eingewandt, sie bewirkten eine „Amerikanisierung“ des Wahlkampfes. ${ }^{43}$ Damit sind insbesondere der Trend, mit bestimmten Personen zu werben, und die Auseinandersetzungen zwischen den Kontrahenten à la „Kanzlerduell“ gemeint, aber auch Finanzierungspraktiken. ${ }^{44}$ Die hiermit geltend gemachte Kritik beruht allerdings eher auf einer begrifflichen Unschärfe. Nicht jede Form der Personalisierung ist abzulehnen. So ist ein gewisser Grad sogar unbedingt notwendig, denn wie sollten politische Vorgänge durch die Öffentlichkeit kontrolliert werden, wenn es keine Personen gibt, die mit politischen Entscheidungen in Verbindung gebracht werden können? Eben diese kritische Partizipation durch die Öffentlichkeit soll durch ein Wahlsystem gefördert werden, in dem die zukünftigen Repräsentanten sich dem Bürger in einem weitgehend offenen Wettbewerb der Ideen vorstellen müssen.

Einhergehend mit der verstärkten Personalisierung könnte die Finanzierung des Wahlkampfes und der Kandidaten gewisse Legitimationsprobleme mit sich bringen. Denn durch Vorwahlen verlängert sich zwangsläufig die Zeitspanne, in der die Kandidaten um die Stimmen ihrer Wähler kämpfen müssen. Die Kosten für die Parteien würden ansteigen, sofern diese zu einer Umschichtung der Wahlkampfgelder zugunsten der Vorwahlen nicht bereit wären. Auch könnte der finanzielle Bedarf einzelner Kandidaten verstärkt von privaten Akteuren gedeckt werden und eine Abhängigkeit zu den spendenden Interessengruppen entstehen, die sich der Öffentlichkeit entzieht. ${ }^{45}$ Hier bedarf es klarer rechtlicher Regelungen, die einer solchen Abhängigkeit von - meist nicht uneigennützigen - Geldgebern entgegenwirken und eine weitgehende Chancengleichheit der Kandidaten im Vorwahlkampf sicherstellen.

Widerstand gegen die Neuerungen, die das Personen-Verhältnis-Wahlsystem mit sich bringt, wird nicht zuletzt von Seiten altgedienter, mittlerer Parteieliten zu erwarten sein ${ }^{46}$,

42 BVerfGE 95, S. 335, S. 354.

43 Vgl. zum Beispiel Ulrich von Alemann / Christoph Strünck, Die Weite des politischen Vor-Raumes - Partizipation in der Parteiendemokratie, http://www.phil-fak.uni-duesseldorf.de/politik/Mitarbeiter/ Alemann/aufsatz/99_alemann-struenck_partzipation.pdf, 1999, S. 11.

44 Vgl. Jürgen Dittberner, a.a.O., S. 17.

45 Vgl. Sven T. Siefken, a.a.O., S. 543, S. 545.

46 Vgl. Andreas Kießling, a.a.O., S. 36. 
denn es bleibt nicht aus, dass innerparteiliche Machtpositionen entkräftet oder zumindest durchmischt werden. ${ }^{47}$ Eine lange Karriere im Dienste der Partei dürfte nicht mehr eine solch wichtige Referenz für ein politisches Amt sein, wie dies bisher der Fall war. Gegen solche Widerstände sprechen Befunde der Potsdamer Parteimitglieder-Studie, nach denen die Funktionsträger in den Parteien keineswegs bemüht sind, „ihre angeblichen Privilegien gegen einen zu großen Einfluß der passiven Mehrheit zu verteidigen “48. Hinzu kommt, dass die herkömmliche Parteimitgliedschaft ja keineswegs gänzlich aufgelöst, sondern vielmehr nur durch die Parteiregistrierung ergänzt wird, die der Öffentlichkeit und Allgemeinheit von Parteien Rechnung trägt. Zwei Drittel der Abgeordneten werden zudem weiterhin über die Parteilisten besetzt, so dass die Parteien allzu einseitige Direktkandidatenaufstellungen wieder auszugleichen vermögen.

Die Wirkung der innerhalb des hier vorgeschlagenen Personen-Verhältnis-Wahlsystems erstrebten Reformen würde sich nur langsam entfalten. Auf die Dauer sind aber wertvolle Veränderungen zu erwarten. Dies gilt insbesondere für die personellen und sachlichen $\mathrm{Zu}$ gangsmöglichkeiten, aber auch für die Attraktivität von Politik. Interesse ist der erste Schritt zur Partizipation.

47 Vgl. Sven T. Siefken, a.a.O., S. 548; Gerd Mielke, a.a.O., S. 47.

48 Malte Lübker, Mitgliederentscheide und Urwahlen aus Sicht der Parteimitglieder: empirische Befunde der Potsdamer Mitgliederstudie, in: ZParl, 33. Jg. (2002), H. 4, S. 716 ff., S. 738.

\title{
Anreiz für Bürger, Entlastung für Politiker? Zur Bündelung von Wahlterminen
}

\author{
Michael Sitsen
}

Die Diskussion, ob Bundes- und Landtagswahltermine zusammengelegt werden sollen, kommt immer wieder auf ${ }^{1}$. Erneut geben dazu die aktuellen Überlegungen der Großen Koalition, die Wahlperiode des Bundestags von vier auf fünf Jahre zu verlängern, Anlass ${ }^{2}$.

1 Kommission Verfassungsreform, BR-Drucks. 360/92, S. 16; Claus Asmussen / Ulrich Eggeling, Empfehlungen des Bundesrates zur Stärkung des Föderalismus in Deutschland und Europa, in: VerwArch, 84. Jg. (1993), S. 230, S. 249; Thomas Friedrich, Gemeinsamer Termin für die Wahl zum Bundestag und zu den Landtagen in den neuen Bundesländern im Jahr 1994?, in: ZRP 1993, S. 363; Klaus von Beyme, Zusammenlegung von Wahlterminen: Entlastung der Wähler - Entlastung der Politiker?, in: ZParl, 23. Jg. (1992), H. 2, S. 339; Hartmut Maurer, Die Verlängerung und Anpassung der Wahlperioden in Bund und Ländern, in: JuS 1983, S. 45; Leitantrag des Bundesvorstandes der Jungen Union an den Deutschlandtag 2004 in Oldenburg, unter der Überschrift „Vordenken. Deutschland entrümpeln“: es wird angeregt, überall fünf Jahre und die Landtagswahlen gebündelt in der Mitte der fünfjährigen Wahlperiode des Bundestags abzuhalten; Antrag der FDP/DVP an den Landtag von Baden-Württemberg vom 11. Juni 1992, LT-Drucks. 11/32.

2 Im November 2005 fand dazu ein Treffen der Fraktionsvorsitzenden von Union und SPD statt; vgl. Presseartikel unter http://www.stern.de/politik/ausland/550727.html?eid=549015\&nv=cb. 